\title{
Multifractal Detrended Cross-Correlation Analysis of Electricity and Carbon Markets in China
}

\author{
Shaohui Zou $\mathbb{D I D}^{1,2}$ and Tian Zhang $\mathbb{D}^{2}$ \\ ${ }^{1}$ School of Management, Xi'an University of Science and Technology, Xian 710054, China \\ ${ }^{2}$ Energy Economy and Management Research Center, Xian University of Science and Technology, China
}

Correspondence should be addressed to Tian Zhang; 2274540847@qq.com

Received 3 January 2019; Revised 24 March 2019; Accepted 21 April 2019; Published 14 May 2019

Academic Editor: Gen Q. Xu

Copyright (c) 2019 Shaohui Zou and Tian Zhang. This is an open access article distributed under the Creative Commons Attribution License, which permits unrestricted use, distribution, and reproduction in any medium, provided the original work is properly cited.

With the development of carbon market, the complex dynamic relationship between electricity and carbon market has become the focus of energy research area. In this paper, we applied a new developed multifractal detrended cross-correlation analysis method to investigate the cross-correlation and multifractality between electricity and carbon markets. We analyze the daily return of electricity and carbon prices over a period of 6 years to do the research. The results show that, firstly, we find that there is a strong negative correlation between domestic carbon price and electricity price and a significant cross-correlation between the return series of electricity and carbon markets. Secondly, through multifractal detrended fluctuation analysis, it is proven that there are obvious multifractal characteristics in the return series of electricity and carbon markets, and the results of traditional linear analysis are unreliable. We also find that, based on multifractal detrended cross-correlation analysis, the law cross-correlation between electricity and carbon markets exists significantly. The long-range correlation of small fluctuations and large fluctuations and the fat tail distribution of return series are the reasons for the formation of multifractality.

\section{Introduction}

Since the industrial revolution in Western countries, the rapid development of global economy, the rapid growth of world population, and the sharp increase of fossil energy consumption have all led to the continuous rise of greenhouse gas concentration. The most important greenhouse gas is carbon dioxide, which seriously restricts the sustainable development of human society. According to the Global Carbon Project's Global Carbon Budget Report 2017, China's carbon emissions have accounted for $26 \%$ of global carbon emissions. In order to actively respond to climate change and energy conservation and emission reduction, the Chinese government has started the construction of regional carbon emission trading market since 2013. According to statistics, the cumulative quota turnover of carbon emissions trading pilot projects in China has reached million tons of carbon dioxide equivalent and about 4.5 billion yuan by September 2017 since the start of the pilot project. With the announcement of the National Development and Reform Commission (NDRC) on the "National Carbon Emission Trading
Market Construction Plan (Electricity Generation Industry)" (referred to as the "Construction Plan"), China's carbon market was officially launched on December 19 through the NDRC press conference. According to the construction plan, the coverage of the national carbon market is currently only included in the electricity industry.

Carbon market construction is to give full play to the decisive role of the market in resource allocation. Carbon market is a man-made market. The core of its operation mechanism is to restrict the emission of greenhouse gases (carbon dioxide or carbon dioxide) through the implementation of laws and regulations, which makes the allowable emission of carbon quota (emission rights), that is, the subject matter of trading, scarce and also makes the carbon emission rights have value or commodity attributes. Electric electricity industry is an energy conversion industry. The main way to develop low-carbon energy is to reduce the use of primary high-carbon energy (fossil energy) or to improve the efficiency of high-carbon energy. When the total carbon emission target of the industry is fixed, it is necessary to improve the electricity generation structure and improve the 
electricity generation efficiency. In the decades of reform and opening up, especially in the past decade, the electric electricity industry has made remarkable achievements in reducing carbon emissions from electricity generation efficiency and new energy technologies. According to the preliminary analysis of ITU, carbon dioxide emissions per unit of thermal electricity generation in 2016 were about 822 g/kWh, falling by $21.6 \%$ since 2005 . By the end of 2016 , the installed capacity of nonfossil energy electricity generation in China will reach 570 million kilowatts, accounting for about $35 \%$ of the total installed capacity. During the decade from 2006 to 2016, the electricity industry has reduced carbon dioxide emissions by about 9.4 billion tons. It is increasingly difficult to further tap the technical potential of carbon emission reduction in the electricity industry, and it is difficult to sustain deep carbon reduction through mandatory technical standards. Therefore, through the construction of the carbon market, promoting the formation of new technology emission reduction potential has become one of the important purposes of the carbon market [1]. Carbon dioxide emission reduction is valuable, and it is a concept that needs to pay economic costs. It will obviously affect the producers and operators of enterprises, further improve the understanding of low-carbon development of enterprises, optimize the construction, production, and operation activities of electricity enterprises, and improve the level of lowcarbon development. At the same time, the carbon price formed by the carbon market is conducive to transmitting the low-carbon development cost to the social level through the electricity market, thus promoting the low-carbon development of the whole society. The construction plan also mentions that the construction of carbon market should "strengthen coordination with relevant policies and measures such as electricity system reform, total energy consumption, strong "double control" and air pollution prevention and control in accordance with the overall arrangement of supplyside structural reform." Therefore, it is of great significance to study the relationship between carbon and electricity markets.

\section{Literature Review}

As far as we all know, many previous researches have indicated that there exist multifractality and long-range correlations in financial markets, and the financial time series are extremely complex and have nonlinear properties [25]. In the prior works, based on the theory of single fractal and multifractal, many methods have been developed to quantify the autocorrelation and cross-correlation behavior of financial markets. WANG Yu-dong et al. [6] examined the relationship between A-share market and B-share market in China. Qualitatively, through statistical analysis, they found that there was a significant correlation between the return series of A-share market and B-share market in China. Quantitatively, using defuzzing cross-correlation analysis, they found that these cross-correlations had strong multifractality in the short term and weak multifractals in the long term. GU Rong-bao et al. [7] have found "rolling windows" in generalized Hurst exponents obtained from three periods divided by two Gulf Wars so that WTI and Brent crude oil returns possess different properties above and below the windows, respectively. Alvarez-Ramirez et al. analyzed the autocorrelation of international crude oil price by using the method of elimination trend fluctuation analysis (DFA). They pointed out that the fluctuation of crude oil market price was dynamic and had fractal characteristics $[8,9]$. Effective market hypothesis has more stringent assumptions that make it unable to explain many financial phenomena comprehensively and effectively. Researchers began to question the rationality of the effective market hypothesis. Therefore, the fractal theory proposed by Mandelbrot has gradually become a new direction to study financial markets in recent years, and the fractal market hypothesis proposed by Peters has become a specific application of fractal theory in financial markets [10-12]. Lo [13] and Hiemstra and Jones [14] used ARFIMA model proposed by Granger [15] in 1980 to empirically test the long-term memory characteristics of emerging stock markets such as the United States and South Korea. The results show that there are long-term memory characteristics in stock markets. Wang et al. [16] studied the change of WTI crude oil market efficiency with time based on DFA and thought that the crude oil market became more and more efficient with time. In order to study the multifractal characteristics of the interactive correlation between two nonstationary time series, Zhou combined MFDFA and DCCA to propose a multifractal elimination trend interactive correlation analysis method (MF-DCCA) [17]. Wang et al. [18] also used DFA and DCCA to study the autocorrelation and interactive correlation of WTI crude oil spot and future yield series. In addition, multifractal spectrum analysis is a multifractal analysis method that describes the internal complexity and local characteristics of time series based on fractal statistical parameters such as mass index, singular index, and multifractal spectrum. It has an important application in the study of financial market complexity [19]. Serletis et al. used R/S method to calculate the Hurst index of the oil price series and proved that the WTI oil market has long-term memory characteristics [2023]. Chen et al. used MFDFA and multifractal spectrum to analyze the multifractal characteristics and causes of the futures price return series of metals and agricultural products in China. The results show that long-term memory is the main reason for the existence of multifractal characteristics in the market $[24,25]$.

Based on the previous research, this paper mainly uses multifractal analysis method to study the correlation between domestic electricity and carbon markets. Our novel contributions are threefold. First, ours is the first study to apply the MF-DCCA method in the empirical analysis of multifractal cross-correlations between electricity and carbon markets in China. Second, we used the rolling windows method to study the dynamics of short-term cross-correlations between electricity and carbon markets in China. Third, our empirical results, to some extent, indicate that multifractal characteristics exist in the return series of electricity and carbon markets. Electric Utilities Sub-Industry Index (EUI) price published by WIND represents the price of electricity market, and the price of carbon emission trading in Shanghai (SHE) 
represents the price of carbon market. Firstly, the correlation test statistics are used to qualitatively illustrate the crosscorrelation between the price-return series of these two markets, pointing out that the cross-correlation has multifractal characteristics, and then the multifractality methods such as MF-DCCA and multifractal spectrum analysis are used to quantitatively measure the multifractal strength of the crosscorrelations between these two markets. Compare the risk of the markets and explore the source of the multifractal relationship between the markets. This study will help people to understand the relationship between domestic electricity and carbon markets, the volatility characteristics, and risk size of the market more deeply and provide some theoretical proposals for market participants.

This paper is organized as follows. Section 3 mainly focuses upon the description of MF-DFA and MF-DCCA. Section 4 describes the data of two markets of returns. Section 5 provides the empirical analysis. Section 6 discusses the empirical results based on rolling windows method and Section 7 presents the conclusion of this paper.

\section{Methodology}

Zhou [17] combines MFDFA method with DCCA method proposed by Podobnik [26] and proposes MF-DCCA method, which is mainly used to study the correlation and multifractal characteristics of two nonstationary series, thus providing a new method for this study. We suppose that there are two time series $x_{i}$ and $y_{i}$; we have $i=1,2,3 \ldots, \ldots L$, when $L$ is the length of the two time series. We will introduce MFDCCA method by the following steps.

The first step is determining the profile of time series:

$$
\begin{aligned}
& X(i)=\sum_{n=1}^{i}(x(n)-\bar{x}), \\
& Y(i)=\sum_{n=1}^{i}(y(n)-\bar{y}),
\end{aligned}
$$

in which $\bar{x}=(1 / L) \sum_{i=1}^{L} x(i), \bar{y}=(1 / L) \sum_{i=1}^{L} y(i)$.

The second step is dividing the profile time series $x_{i}$ and $y_{i}$ into $L_{s}=\operatorname{int}(L / s)$ nonoverlapping segments of equal length $s$. Since $\mathrm{N}$ is usually not an integer multiple of $s$, in order not to neglect the last series, the segmentation process is repeated from the tail of the series to obtain $2 L s$ subseries.

The third step is that the local trends $X^{u}(i)$ and $Y^{u}(i)$ for each segment $u\left(u=1,2,3 \ldots, 2 L_{s}\right)$ are evaluated by leastsquares fits of the time series, followed by fitting $2 L s$ subseries with $m$-order polynomials, eliminating trend covariance

$$
\begin{aligned}
F^{2}(s, u)= & \frac{1}{s} \sum_{i=1}^{s}\left|X((u-1) s+i)-\widetilde{X}_{u}(i)\right| \\
& \cdot\left|Y((u-1) s+i)-\tilde{Y}_{u}(i)\right|,
\end{aligned}
$$

when $u=1,2,3 \ldots, L_{s}$;

$$
\begin{aligned}
F^{2}(s, u)= & \frac{1}{s} \sum_{i=1}^{s}\left|X\left(L-\left(u-L_{s}\right) s+i\right)-\widetilde{X}_{u}(i)\right| \\
& \cdot\left|Y\left(L-\left(u-L_{s}\right) s+i\right)-\widetilde{Y}_{u}(i)\right|
\end{aligned}
$$

when $u=L_{s}+1, L_{s}+2, L_{s}+3 \ldots, 2 L_{s}$. Therefore, the trends $\widetilde{X}_{u}(i)$ and $\widetilde{Y}_{u}(i)$ denote the fitting polynomial with order $m$ in each part $u$.

The fourth step is calculating $q$ th-order detrended covariance function

$$
\begin{aligned}
& F_{q}(s)=\left\{\frac{1}{2 L_{s}} \sum_{u=1}^{2 L_{s}}\left[F^{2}(s, u)\right]^{q / 2}\right\}^{1 / q}, \quad q \neq 0 \\
& F_{0}(s)=\exp \left\{\frac{1}{4 L_{s}} \sum_{u=1}^{2 L_{s}} \ln \left[F^{2}(s, u)\right]\right\}, \quad q=0 .
\end{aligned}
$$

The fifth step is that, for each fixed value of $q$, determine the scaling behavior of covariance fluctuations by observing $\log -\log$ plots $F_{q}(s)$. If there is a long-range electricity law interaction between two series $x_{i}$ and $y_{i}$, then, for a large value of $s$, we have

$$
F_{q}(s) \propto s^{H_{x y}(q)}
$$

$H_{x y}(q)$ represents the cross-correlation exponent, describing the electricity-carbon relationship between two time series. Especially, when the two series $x_{i}$ and $y_{i}$ are identical, then MF-DCCA is simplified to MF-DFA. When $q$ is equal to 2, MF-DCCA is the standard of the DFA. Besides, if the cross-correlation scaling exponent $H_{x y}(q)$ changes with $q$, the cross-correlation between the two sequences is multifractal. If the cross-correlation scaling exponent $H_{x y}(q)$ is not dependent on $q$, the cross-correlation between the two sequences is monofractal. If the cross-correlation scaling exponent $H_{x y}(q)>0.5$, the cross-correlations between the return fluctuations of the two series related to $q$ are long-range persistent, which means that one rising price is likely to follow another rising price. If the cross-correlation scaling exponent $H_{x y}(q)<0.5$, the cross-correlations between the return fluctuations of the two series are antipersistent, which means that one rising price is likely to follow another falling price. If the cross-correlation scaling exponent $H_{x y}(q)=0.5$, there are no cross-correlations between the return fluctuations of the two series related to $q$. In addition, for positive $q$, $H_{x y}(q)$ describes the scaling behavior of intervals with large fluctuations. On the contrary, for negative $q, H_{x y}(q)$ describes the scaling behavior of the segments with wavelet fluctuations [2].

$\Delta H$ can be used to measure the degree of multifractality between two series, which is expressed as

$$
\Delta H=H_{\max }(q)-H_{\min }(q)
$$

Obviously, the higher variability of $H(q)$ is, the richer multifractality will be. Therefore, the larger $\Delta H$ is, the 


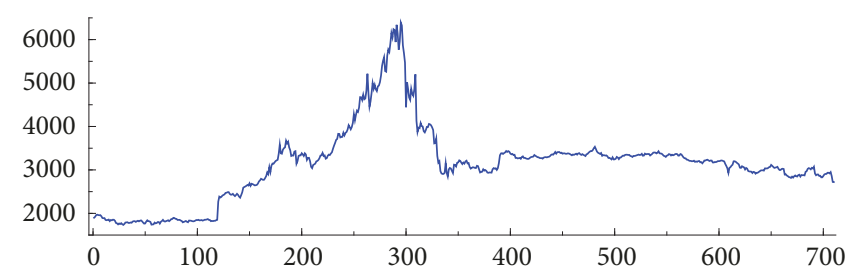

- EUI

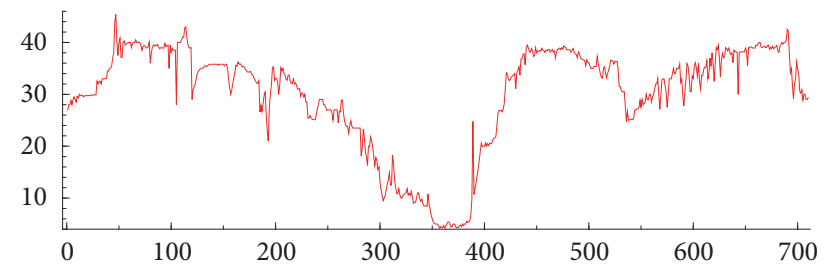

- SHE

FIGURE 1: Daily prices of electricity and carbon markets (the red and blue lines represent the price series of electricity and carbon markets, resp.).

stronger the degree of multifractality will be, and then the market is becoming more inefficient.

It is pointed out that there is the following relationship between the $q$ th-order cross-correlation exponent $H_{x y}(q)$ and the multifractal quality exponent (Renyi exponent) $\tau_{x y}(q)[27]:$

$$
\tau_{x y}(q)=q H_{x y}(q)-1
$$

If the scaling exponent formula $\tau_{x y}(q)$ is linear to $q$, the cross-correlations of the series are considered to be monofractal. If the scaling exponent formula $\tau_{x y}(q)$ is not linear to $q$, the cross-correlations of the two time series are considered to be multifractal. Through Legendre transformation, the following relations can be obtained:

$$
\begin{aligned}
\alpha & =H_{x y}(q)+q H_{x y}^{\prime}(q) \\
f_{x y}(\alpha) & =q\left(\alpha-H_{x y}(q)\right)+1
\end{aligned}
$$

In the MF-DFA method, $\Delta h=h_{\max }(q)-h_{\text {min }}(q)$ can be used to describe the degree of multifractality. The greater $\Delta h$ is, the stronger the multifractality will be, which indicates a large fluctuation in the market. The multifractal spectrum $f_{x y}(\alpha)$ describes the singularity content of the time series. [4] In addition, the width of multifractal spectrum reflects the size of market fluctuation. The larger $\Delta \alpha$ is, the more uneven the distribution of time series is and the stronger the multifractal character is. $\Delta \alpha$ is expressed as

$$
\Delta \alpha=\alpha_{\max }-\alpha_{\min }
$$

\section{Indicators and Data Sources}

At present, China's carbon market is still dominated by regional markets, and there is no unified and perfect carbon emission trading market. Since 2013, China has launched seven carbon trading pilot projects in Beijing, Tianjin, Shanghai, Chongqing, Guangdong, Hubei, and Shenzhen to explore the establishment of carbon trading mechanism. This paper chooses Shanghai carbon emission trading price (SHE) data, one of the seven pilot exchanges of China's carbon emission rights. On one hand, the article is limited in length; on the other hand, among the seven earliest carbon exchanges in China, the price of Shanghai Carbon Exchange fluctuates greatly and the trading volume is in the forefront, which is representative. In this study, one main carbon price (Shanghai carbon emission trading price (SHE)) is selected. The daily closing price of carbon used in this study is available from Shanghai Environment and Energy Exchange (http://www.cneeex.com/). Electric Utilities SubIndustry Index (EUI) price is selected to represent the electricity price, which is available from Wind database. The data was collected from 28 November 2013 till 12 October 2018. After eliminating the nonmatching missing data, we get the length of the pair of series SHE \& EUI, which consists of 709 data points. We define the daily price return $r_{t}$ and the natural logarithmic difference of the daily closing price $P_{t}$ as $r_{t}=\ln \left(P_{t}\right)-\ln \left(P_{t-1}\right)$. Figure 1 presents the curve of daily prices of carbon and electricity markets. The daily returns of all time series are given in Figure 2, which shows the presence of clusters of small and large fluctuations.

From Figure 1, we can see that there is a strong negative correlation between domestic carbon price and electricity price. At the same time, it is also found that both of them show abnormal characteristics in some time periods, but with the passage of time, the abnormal characteristics become more and more obscure. In the long run, the price fluctuation of electricity market is similar to an open-down quadratic parabola, while the trend of carbon price is similar to an open-up quadratic parabola.

As can be seen from Figure 2, the two daily returns show the characteristics of violent fluctuation and volatility agglomeration effect, and the volatility of the electricity 

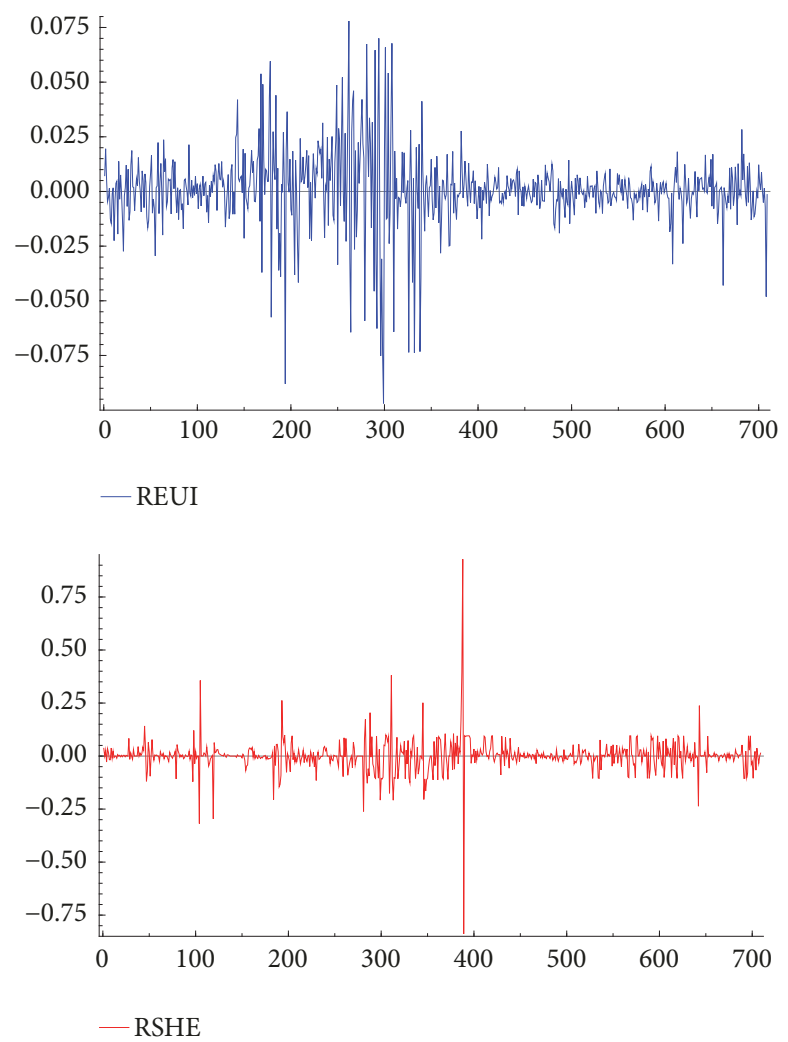

FIGURE 2: Returns of electricity and carbon time series.

market is larger than that of the carbon market, indicating that the electricity market is more effective and responds more quickly to relevant information.

In order to carry out multifractal analysis of the daily returns of electricity price and carbon price, firstly, we use traditional statistical methods to test their statistical characteristics. The descriptive statistics summarize the general behavior of all two time series, which is given in Table 1 .

As can be seen from Table 1, the average return of the two series is $1.2011 \%$ and $8.2226 \%$, respectively, which means that the average return of electricity market is larger than that of carbon market. The skewness of carbon market is negative, while that of electricity market is positive, and the skewness of electricity market is much larger than that of carbon market, which indicates that the return of electricity market is more asymmetric. The peaks of the two return series are 52.18809 and 9.733704, respectively, which indicates that the distribution of the two series has peak characteristics and does not obey normal distribution. Judging from J-B test, both return series reject the normal hypothesis at the significant level of $1 \%$, showing the characteristics of "peak and heavy tail."

\section{Empirical Results}

5.1. Cross-Correlations Test. This paper used cross-correlation statistic $Q_{c c}(m)$ to study the nonlinear cross-correlations between electricity and carbon markets. Here, for two series $x_{i}$ and $y_{i}$, we have $i=1,2,3 \ldots, \ldots L$; the test statistic and the cross-correlation function are expressed as the following two functions:

$$
\begin{aligned}
Q_{c c}(m) & =N^{2} \sum_{i=1}^{m} \frac{X_{i}^{2}}{N-i}, \\
X_{i} & =\frac{\sum_{k=i+1}^{N} x_{k} y_{k-i}}{\sqrt{\sum_{k=1}^{N} x_{k}^{2} \sum_{k=1}^{N} y_{k}^{2}}}
\end{aligned}
$$

If the cross-correlation statistic $Q_{c c}(m)$ approximately obeys the chi-square distribution with degree of freedom $\mathrm{m}$, there are no cross-correlations between two time return series; otherwise, the cross-correlations are significant at a special significance level [4]. The cross-correlation test is carried out on six combinations of bivariate time series and the critical value of the $\chi^{2}(m)$ distribution at the $5 \%$ level of significance for the degrees of freedom varying from 1 to 1000.

Figure 3 shows the log-log plot of cross-correlation statistic $Q_{c c}(m)$, in which the degree of freedom $m$ is taken from 1 to 1000 as the control. The critical value of chisquare distribution at $95 \%$ confidence level is also given in Figure 3. Obviously, the cross-correlation statistic $Q_{c c}(m)$ deviates from the critical value of chi-square distribution with degree of freedom $m$. Therefore, the original hypothesis of no cross-correlation is rejected, which indicates that there is an interactive correlation between the carbon market and the electricity markets.

To make the results more accurate, in this paper, we have also used another statistical test method proposed by 
TABLE 1: Descriptive statistics for the returns of carbon and electricity market time series.

\begin{tabular}{lcc}
\hline Statistics & RSHE & REUI \\
\hline Mean & 0.012011 & 0.082226 \\
Median & 0 & 0.074004 \\
Maximum & 92.84613 & 7.794301 \\
Minimum & -83.87325 & -9.690959 \\
Std. Dev. & 7.794822 & 1.758069 \\
Skewness & 0.844039 & -0.564978 \\
Kurtosis & 52.18809 & 9.733704 \\
Jarque-Bera & 71559.32 & 1377.22 \\
Probability & 0 & 0 \\
Sum Sq. Dev. & 43017.55 & 2188.291 \\
Observations & 709 & 709 \\
\hline
\end{tabular}

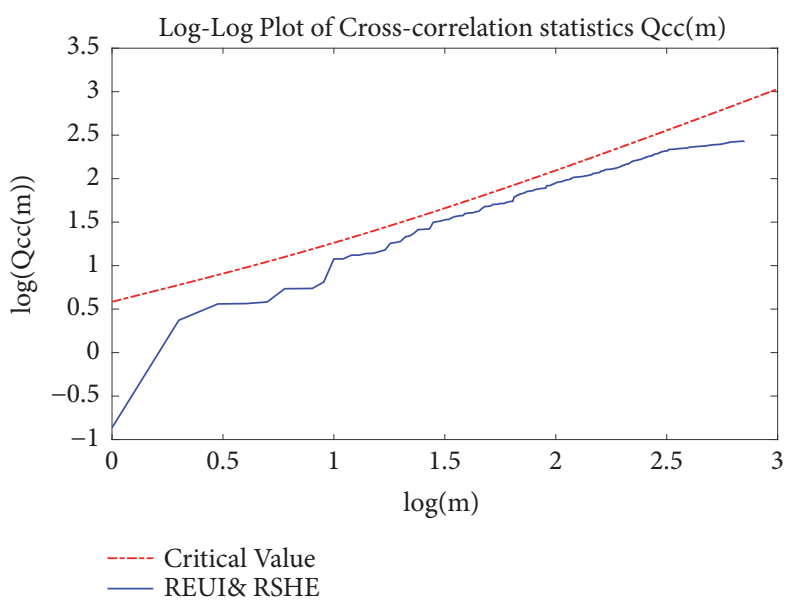

FIGURE 3: Log-log plot of test statistics $Q_{c c}(m)$ (the blue and red lines represent the pairs of REUI \& RSHE and critical value, resp.).

Podobnik et al. to quantify the level of cross-correlations between two time series, which is defined as the ratio between function of detrended covariance $F_{D C C A}^{2}$ and the detrended variance $F_{D F A}^{2}$. The difference between the original walk and the local trend is "detrended walk." According to the "detrended walk," we calculate the covariance of the residuals in each box [28]:

$$
\begin{aligned}
f_{D C C A}^{2}(n, i) & \equiv \frac{1}{(n-1) \sum_{k=i}^{i+n}\left(R_{k}-\widetilde{R}_{k, i}\right)\left(R_{k}^{\prime}-\widetilde{R}_{k, i}^{\prime}\right)} \\
R_{k} & \equiv \sum_{i=1}^{k} y_{i}, R_{k}^{\prime} \equiv \sum_{i=1}^{k} \widetilde{y}_{i}
\end{aligned}
$$

For both time series, in each window that starts at $i$ and ends at $i+n$, we define a "local trend $\widetilde{R}_{k, i}$ ". The detrended covariance function is as follows:

$$
\begin{gathered}
F_{D C C A}(n) \equiv \sqrt{\frac{1}{N-n} \sum_{i=1}^{N-n} f_{D C C A}^{2}(n, i)} \\
F_{D F A}(n) \equiv \sqrt{\frac{1}{N-n} \sum_{i=1}^{N-n} f_{D F A}^{2}(n, i)}
\end{gathered}
$$

The formula is as follows:

$$
\rho_{D C C A}=\frac{F_{D C C A(n)}^{2}}{F_{D F A 1(n)} F_{D F A 2(n)}}
$$

In the above formula, the value of $\rho_{D C C A}$ ranges within $-1 \leq \rho_{D C C A} \leq 1$. If $\rho_{D C C A}=0$, there is no cross-correlation between two time series. If $\rho_{D C C A}=1$, it means there is a perfect cross-correlation between two time series and if $\rho_{\text {DCCA }}=-1$, it indicates a perfect anti-cross-correlation existing in the time series. In this paper, we have calculated the cross-correlation coefficient, and Figure 4 shows $\rho_{D C C A}$ for all the bivariate time series for different scale size $n$. From [29], we know that if $\rho_{D C C A}$ (DCCA cross-correlation coefficient) is greater than 0.269 (seen in Table 1 in [29]), we can reject the null hypothesis that there is no crosscorrelation between the two series. However, from Figure 4, we can see that the minimum value of $\rho_{D C C A}$ between carbon and electricity markets is 0.35 , so the two time series have long-range cross-correlation. Because of the finite size of time series, even if there is no cross-correlation, $\rho_{D C C A}$ is not equal to 0 . This cross-correlation coefficient test is used to show the existence of cross-correlation. Therefore, in order to find out whether the cross-correlation is long range or anticorrelation, 


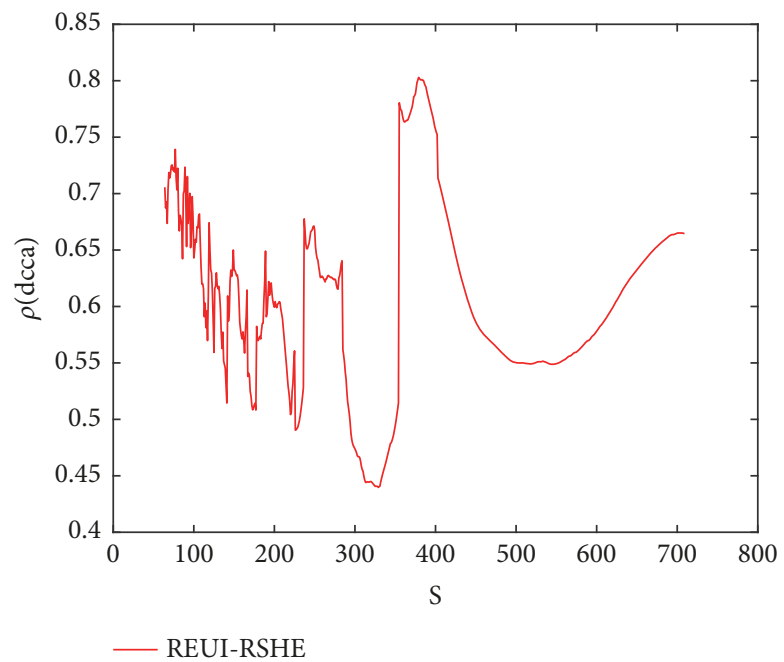

FIGURE 4: The cross-correlation coefficient of REUI and RSHE by detrended cross-correlation analysis.

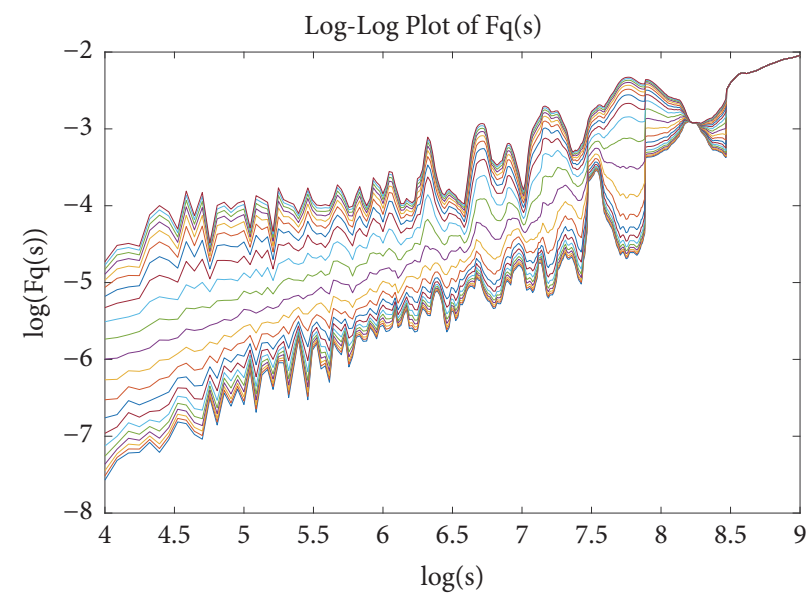

FIGURE 5: Log-log plots of $F_{q}(s)$ versus time scale between carbon and electricity markets.

DCCA method and its variants are needed to be applied to our study.

5.2. Analysis of MF-DCCA Method. Cross-correlation statistics Qcc $(m)$ can only qualitatively test the existence of crosscorrelations between carbon and electricity markets. Multifractal detrended cross-correlation analysis (MF-DCCA) and multifractal spectrum analysis can more quantitatively characterize the characteristics and intensity of the correlation between return series. Therefore, in order to affirm our results obtained above, the MF-DCCA method proposed by Podobnik and Stanley (2008) is quantitatively applied to test the presence of cross-correlation.

According to the multifractal detrended cross-correlation (MF-DCCA) method, Figure 5 shows the log-log plots of the fluctuation function $F_{q}(s)$ versus time scale $s$ between the carbon and electricity markets. As can be seen from Figure 5, for different $q$ values $(q=-10,-8, \ldots, 8,10)$, all curves are approximately linear under large scales, which further shows that there is power-law cross-correlation in the return series. The power-law cross-correlation relationship shows that a great price change in the electricity market is more likely to be followed by a great price change in carbon market, and a large price change in the carbon market is more likely to be followed by a large price change in electricity market.

Table 2 shows the variation of the cross-correlation exponents $\mathrm{H}_{\mathrm{xy}}(q)$ with $q$ from -10 to 10 . It can be seen that $\mathrm{H}_{\mathrm{xy}}(q)$ decreases nonlinearly with the increase of $q$ value, which indicates that the cross-correlation between the two markets has multifractal characteristics. When $q$ is less than or equal to 4, the cross-correlation exponent of carbon market and electricity market (REUI/RSHE) is larger than 0.5 ; when $q$ is greater than or equal to 3 , it is smaller than 0.5 ; it shows that the cross-correlation of sequence wavelet fluctuations is persistent, while the cross-correlation of large fluctuations is antipersistent. From Table 2, we can see that the multifractal strength $\mathrm{H}_{x y}(q)$ of carbon market and electricity market is larger than that of electricity market itself, which indicates that the portfolio of electricity market and carbon market bears greater financial risks.

When $q$ equals 2 , the scaling exponent $H_{x y}(q)$ has an explanation similar to classical Hurst exponent by means 
TABLE 2: Scaling exponents $H_{\mathrm{xy}}(q), H_{\mathrm{xx}}(q)$, and $H_{\mathrm{yy}}(q)$ for return series with $q$ varying from -10 to 10 .

\begin{tabular}{|c|c|c|c|}
\hline$q$ & REUI/RSHE & REUI/REUI & RSHE/RSHE \\
\hline-10 & 0.59386237 & 0.716281715 & 1.000176407 \\
\hline-9 & 0.58539464 & 0.706819522 & 0.99003649 \\
\hline-8 & 0.5753821 & 0.695535404 & 0.977471404 \\
\hline-7 & 0.56341251 & 0.681954121 & 0.961501652 \\
\hline-6 & 0.54894018 & 0.66548597 & 0.940558521 \\
\hline-5 & 0.53124972 & 0.64544136 & 0.911993128 \\
\hline-4 & 0.50943791 & 0.621106456 & 0.871061305 \\
\hline-3 & 0.48249112 & 0.591938227 & 0.808830636 \\
\hline-2 & 0.44967303 & 0.557980322 & 0.709026216 \\
\hline-1 & 0.41146581 & 0.520600915 & 0.554176836 \\
\hline 0 & 0.37033447 & 0.482948373 & 0.374562022 \\
\hline 1 & 0.32976237 & 0.44850466 & 0.241824239 \\
\hline 2 & 0.29267235 & 0.419020515 & 0.162658046 \\
\hline 3 & 0.26071289 & 0.394563717 & 0.114460845 \\
\hline 4 & 0.23417611 & 0.374475676 & 0.08250163 \\
\hline 5 & 0.21248607 & 0.357944623 & 0.059538412 \\
\hline 6 & 0.19478057 & 0.34423255 & 0.042020195 \\
\hline 7 & 0.18023502 & 0.332739874 & 0.028087979 \\
\hline 8 & 0.16816895 & 0.323002463 & 0.016683834 \\
\hline 9 & 0.15805252 & 0.314667064 & 0.007154617 \\
\hline 10 & 0.14948153 & 0.307464868 & -0.000932134 \\
\hline$\Delta \mathrm{H}(q)$ & 0.44438084 & 0.408816847 & 1.001108541 \\
\hline
\end{tabular}

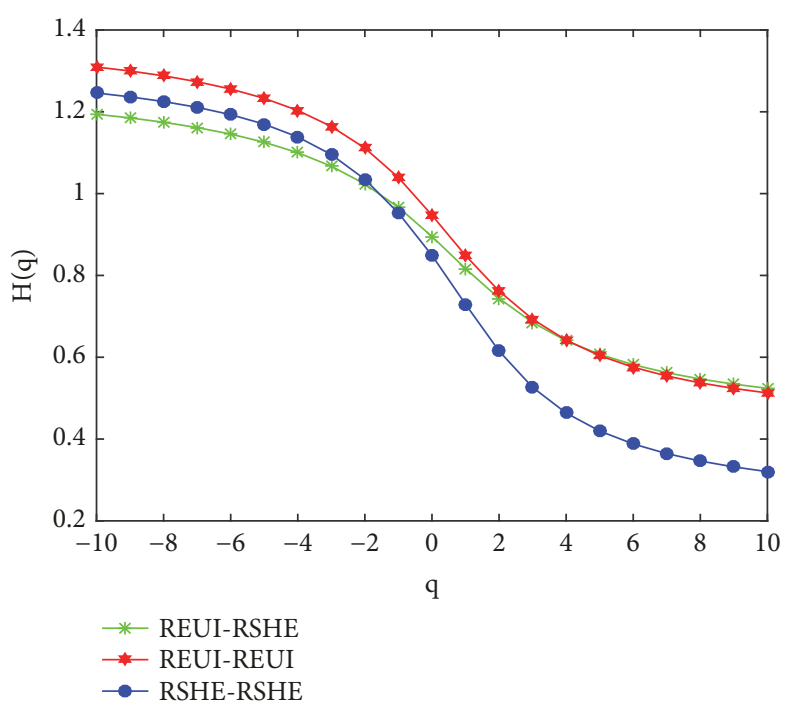

FIGURE 6: $H(q) \sim q$ graph of return series between electricity and carbon markets (the red hexagonal and blue circle curves estimated by MF-DFA method and the green star line estimated by MF-DCCA method).

of MF-DFA method. The cross-correlation between the two series is antipersistent, if the cross-correlation exponents of REUI \& RSHE $\mathrm{H}_{\mathrm{xy}}$ (2) is less than 0.5. When $q=-10$, $H_{x y}(q)$ is significantly higher than 0.5 , indicating that the interaction correlation of extreme wavelet fluctuations is strong and persistent; when $q=10, H_{x y}(q)$ is significantly less than 0.5 , indicating that the cross-correlation of extreme large fluctuations is strong and antipersistent.
Figure 6 shows that the scaling exponents $H_{x y}(q)$ are different with different values of $q$, from which it can be seen that $H_{x y}(q)$ decreases with a change of $q$ value's nonlinearity. We can find that, for REUI \& RSHE, the scaling exponents decrease from 0.59 to 0.14 , indicating that the cross-correlated behavior between electricity and carbon markets shows great multifractal characteristics. Furthermore, we found that the scaling exponents for $q<-3$ are larger than 0.5 , 


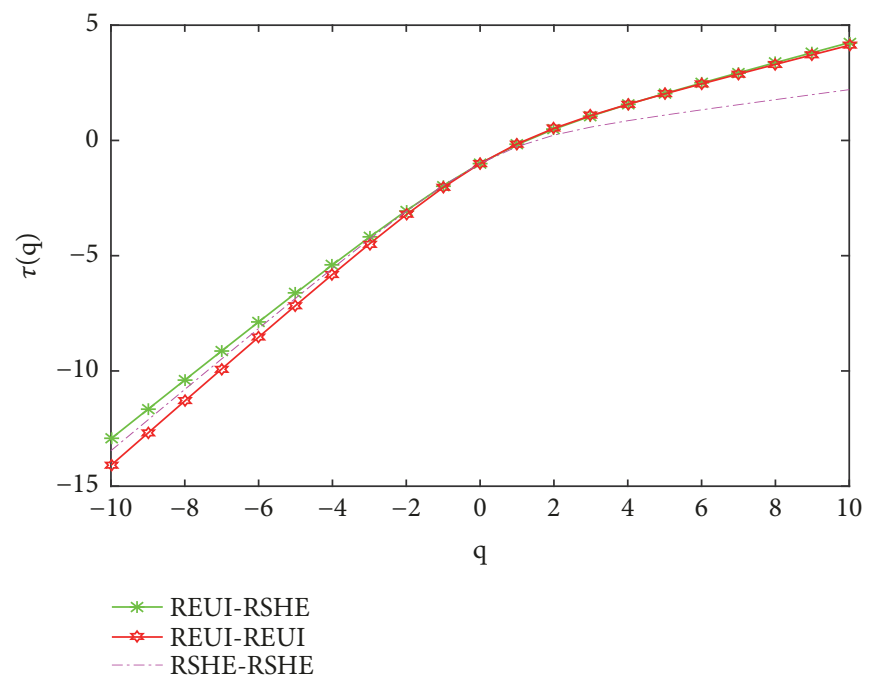

FIGURE 7: $\tau(q) \sim q$ diagram of electricity and carbon markets. The green curve is estimated by MF-DCCA method and the red hexagonal and the pink dotted lines are estimated by MF-DFA method.

which indicates that the cross-correlated behavior of small fluctuations between two markets is persistent. The scaling exponents for $q>-4$ are smaller than 0.5 , demonstrating that the cross-correlated behaviors of large fluctuations are antipersistent. That is to say, the slighter fluctuations of carbon price will lead to small fluctuations in the electricity market.

5.3. Analysis of Multifractal Detrended Fluctuation. For comparison, we also estimate the generalized Hurst exponent $\mathrm{H}(q)$ of each independent series by using the MF-DFA method. The generalized Hurst exponent $\mathrm{H}(q)$ describes the autocorrelation persistence of each sequence. The experimental results are shown by the red hexagonal and the blue circle in Figure 6. If the cross-correlation scaling exponent $\mathrm{H}(q)$ is dependent on $q$, the cross-correlation between the two sequences is multifractal [30]. In our research, we find that $\mathrm{H}(q)$ is not a constant of each underlying series, which means that they are multifractal. Further observation shows that when $q>-4$, the multifractal behavior of large fluctuations in series is antipersistent, while, for $q<-3$, the multifractal behavior of wavelet fluctuations is persistent.

From formula (8), in this paper, we calculate $\tau(q)$ (Renyi exponent). As is shown in Figure 7 , the exponent $\tau(q)$ we calculated from (8) is nonlinearly dependent on $q$, which can also indicate that multifractality does exist in the relationship of electricity and carbon markets.

Figure 7 shows the relationship between quality exponent $\tau(q)$ and $q$ of the two return series. It can be found that they are all increasing nonlinearly, and the two lines of REUI/RSHE and REUI/REUI are close to coincidence, which shows that the nonlinear characteristics of their crosscorrelation are similar, and the multifractalities of crosscorrelation are similar. From the concavity and convexity of the curve, it can be found that the multifractal strength of REUI/RSHE cross-correlation is slightly stronger than that of the series itself.
In order to study the multifractality between electricity and carbon market better, we have to get the multifractal strength by using formula (9). As we all know, only if the system under study is monofractal can the width of the multifractal spectrum be zero. As can be seen from Figure 8, the multifractal spectra of these three series are not a single point, which indicates that multifractality exists not only in the electricity and carbon markets but also in the interrelated market. Figure 8 shows multifractal spectra of the carbon and electricity markets. From Figure 8 , we can see that the width of multifractal spectrum is greater than 0 , which indicates that there is multifractality in the cross-correlation between the two series. When the value of $\alpha$ is large, the multifractal spectrum function $f(\alpha)$ appears to be negative, which indicates that the cross-correlation between markets has greater noise; that is, the market has higher variability. If all $\Delta f(\alpha)=f\left(\alpha_{\min }\right)-f\left(\alpha_{\max }\right)$ are greater than zero, then the multifractal spectrum of the interrelated return series shows a single peak left-hook shape, which indicates that the chance of high price is greater than that of low price, and the return series has a downward trend subsequently.

The calculation results of the width of multifractal spectra $\alpha$ of the return series and the corresponding cross-correlation series are shown in Table 3. Obviously, the cross-correlation multifractal spectrum width of carbon and electricity market return series is significantly larger than 0 , and the crosscorrelation series is significantly smaller than the autocorrelation series, indicating that the cross-correlation multifractal intensity is weaker than the autocorrelation intensity, which may be due to the interaction of external market factors on the asset portfolio including carbon and electricity markets. The adverse effect hedges the risk and reduces the volatility; that is, the risk of the portfolio will be smaller.

5.4. Analysis on the Causes of Multifractality. Usually, there are two main reasons for the formation of multifractality: one is the long-term correlation of large fluctuations and 


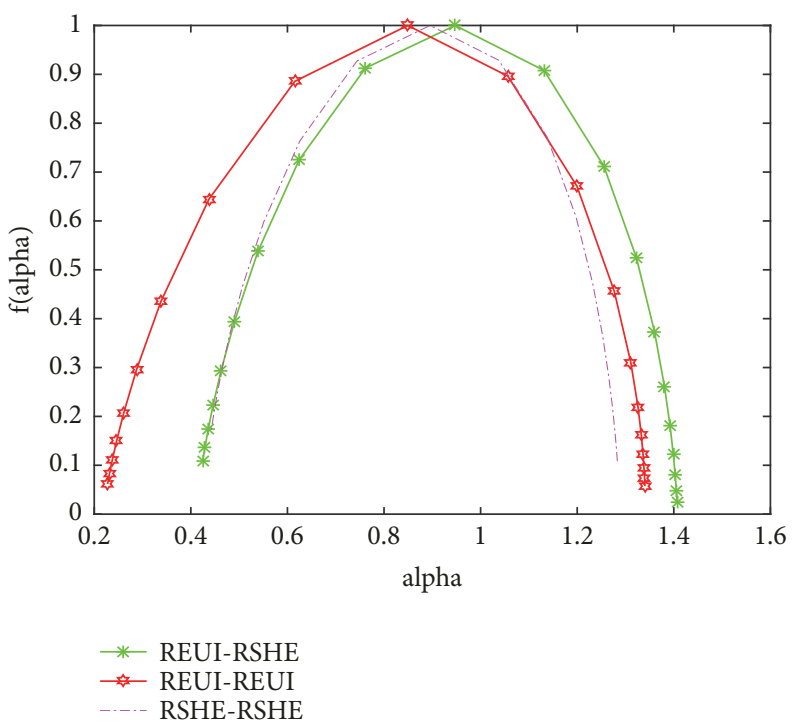

FIGURE 8: Multifractal spectrum for electricity and carbon markets. The red hexagonal and the pink dotted lines are estimated from MF-DFA method, and the green curve is estimated from MF-DCCA method.

TABLE 3: Width of multifractal spectra.

\begin{tabular}{|c|c|c|c|c|c|c|}
\hline & \multicolumn{3}{|c|}{$\Delta \alpha$} & \multicolumn{3}{|c|}{$\Delta H$} \\
\hline & Electricity & Carbon & Cross & Electricity & Carbon & Cross \\
\hline REUI/RSHE & 0.982102099 & 1.1125695 & 0.841346 & 0.7967009 & 0.925635 & 0.669969 \\
\hline
\end{tabular}

small fluctuations; the other is the fat tail distribution of time return series. In order to verify the effect of longrange correlation, we can make a judgment by comparing the multifractal strength of the original series and the shuffled series. In addition, the influence of fat tail distribution on the formation of multifractal can be verified by comparing the multifractal strength of the original series with that of the surrogated series by randomizing the Fourier phase of the original series.

Figure 9 shows the variation of the generalized interactive correlation index $\mathrm{H}(q)$ to $q$ of the original, the shuffled, and the surrogated series. Obviously, the original series, the shuffled series, and the surrogated series of each pair of return series can be clearly distinguished from each other, which shows that the long-range correlation and fat tail distribution are the reasons for the multifractality. For electricity price series (REUI series), $\mathrm{H}(q)$ of surrogated series is closer to $\mathrm{H}(q)$ of original series, which indicates that fat tail distribution is the main reason for the formation of multifractality in electricity market. For carbon price series (RSHE series), $\mathrm{H}(q)$ of the shuffled series is closer to $\mathrm{H}(q)$ of the original series; that is, the long-range correlation is the main reason for the multifractality in carbon market.

\section{Discussion}

6.1. Sample Test of Rolling Windows Method. In 2004, Cajueiro D O and Tabak B M [31] applied the rolling window method to the study the emerging financial markets, in which they proposed the calculation of the Hurst exponent over time using a time window with 4 years of data. On this basis, more and more scholars began to apply the rolling windows method to the study of financial markets. For example, the rolling windows method is introduced to study the effectiveness of China's stock market and the nonlinear relationship between A-share and B-share markets. The window size should be chosen according to the different research questions. In this paper, 100 days are chosen as the length of the rolling window to study the dynamics of shortterm cross-correlations. The results of Figure 10 show that the conclusions of this study are robust.

6.2. Some Important Implications. From Hurst exponents, we can see that, for all binary time series, the value of $H_{x y}(q)$ depends on the value of $q$, which shows that there are obvious multifractal characteristics in carbon and electricity return series. According to the study results of Podobnik and Stanley [26], when $q=2$, the cross-correlation exponent is equal to the average of the individual Hurst exponents for two fractionally autoregressive integrated moving average (ARFIMA) processes sharing the same random noise [32]. Therefore, in our research, we also calculate the average of generalized Hurst exponents, which is defined as the mean of two time series analyzed, respectively, by MF-DFA method (see the following equation).

$$
H_{x y}(q)=\frac{\left[H_{x x}(q)+H_{y y}(q)\right]}{2}
$$

In Figure 11, the pink-dotted line is the average scaling exponents of electricity and carbon markets. As is shown in 

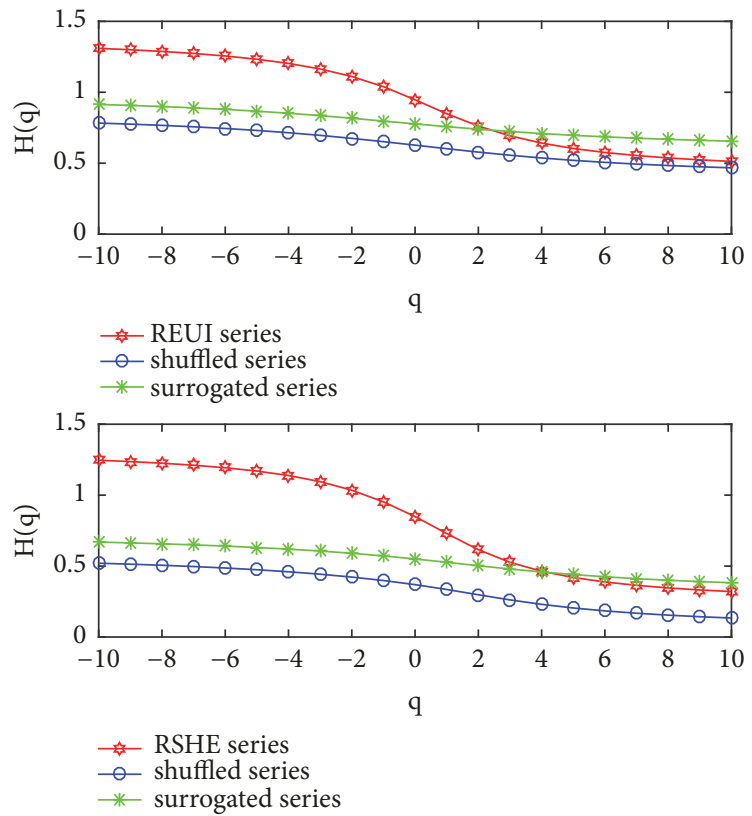

FIGURE 9: Scaling exponents $H(q)$ of the original, shuffled, and surrogated series in electricity and carbon markets.

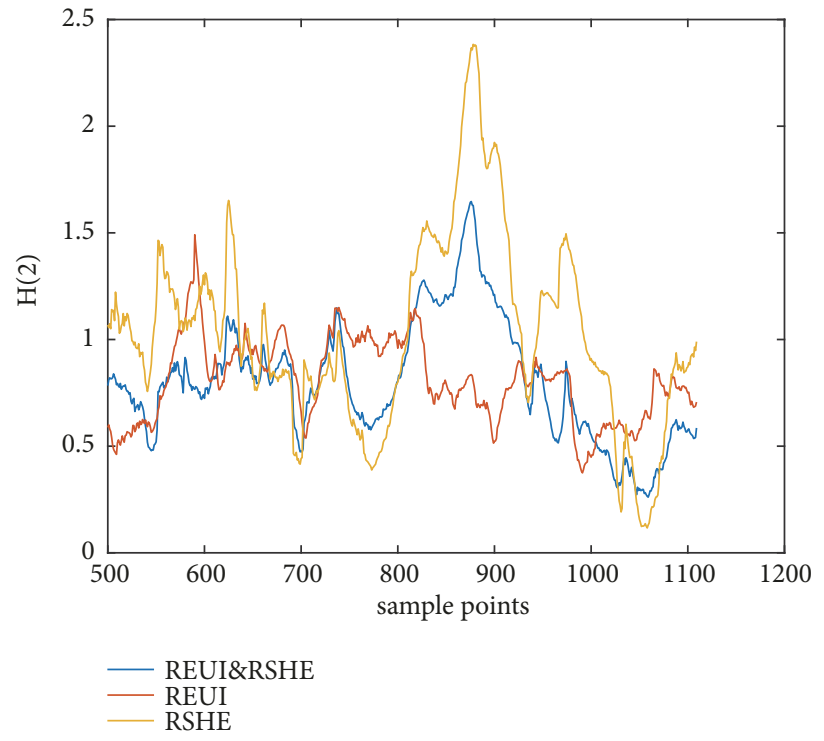

Figure 10: Scaling exponents $H(q)$ versus samples in electricity and carbon markets.

Figure 11, when $q<0$, the cross-correlation exponents are smaller than the average scaling exponents and when $q>0$, the cross-correlation exponents are greater than the average scaling exponents. Meanwhile the exponent of electricity return series is greater than the average scaling exponents for all values of $q$, but the exponent of carbon return series is smaller than the average scaling exponents for all values of $q$.

This paper mainly studies the relationship between China's electricity market and carbon market. Firstly, the evidence from cross-correlation statistical test shows that the existence of cross-correlation is significant at the level of $5 \%$. The empirical results show that the fluctuation of electricity price has a significant impact on carbon price. Therefore, changes in electricity prices will affect the volatility of the carbon market. Secondly, based on the MF-DCCA method, it is found that the correlation of return series between the electricity and carbon markets is multifractal, which indicates that the correlation between the electricity and carbon markets has a nonlinear structure. Therefore, the traditional linear model is not suitable for measuring the complex relationship between electricity and carbon markets. In the study of the relationship between electricity and carbon markets, the interpretation effect of the nonlinear model is more convincing. 


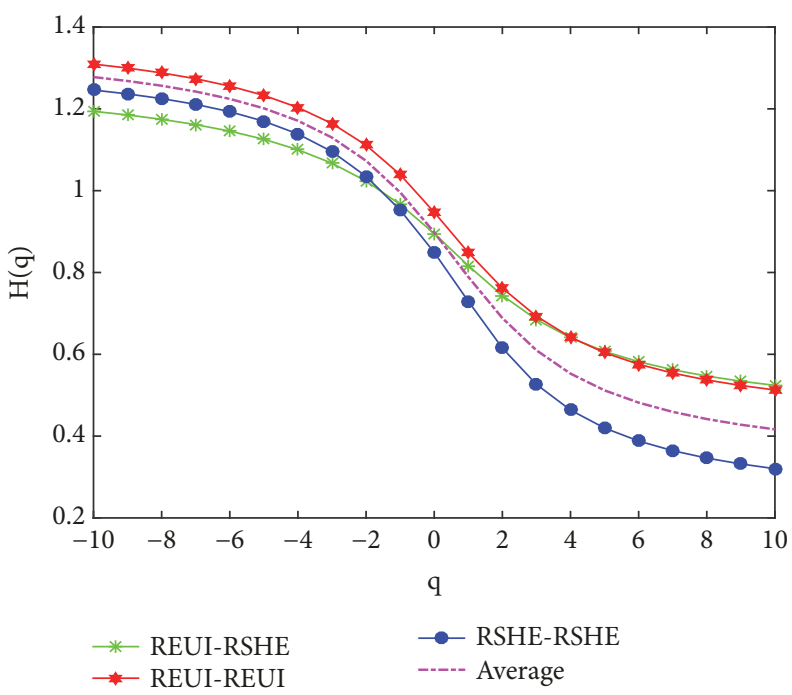

FIGURE 11: The relationship of $H(q)$ versus $q$ with different value of $q$ (the pink dotted line is the average value).

\section{Conclusion}

In this paper, we mainly study the cross-correlation between price return series of domestic electricity carbon markets. Based on basic statistics and cross-correlation test, it is considered that there is a significant cross-correlation between the return series of two markets. MF-DCCA and multifractal spectrum analysis are used to quantitatively analyze the two return series. The results show that the cross-correlation is nonlinear and has multifractal characteristics; and the crosscorrelation between markets is multifractal. Wavelet fluctuation is persistent, while large fluctuation is antipersistent; by comparing the multifractal spectrum width of return series, it is considered that the financial risk of portfolio series is less than that of autocorrelation series. By calculating and comparing the multifractal strength of original series, shuffled series, and surrogated series, it is considered that the long-range correlation of the large fluctuation and the small fluctuation and the fat tail distribution of the return series are the reasons for the formation of the multifractality. This study reveals that although carbon price and electricity price are negatively correlated for a long time under normal circumstances, the relationship is nonlinear and dynamic and has complex multifractal characteristics. The relationship will show different trends with the changes of domestic political and economic situations in different periods, and the intensity of correlation will also change with the changes of time. These results will help to fully understand the complexity of price fluctuations in the two markets and provide valuable references for effective measurement and prevention of market risks and investment decisions.

China is a large country of carbon emissions. In 2016, domestic carbon emissions accounted for $1 / 3$ of the total carbon emissions of the world. Although the Kyoto Protocol does not mandate emission reduction tasks, for the sustainable development of the global economy, China also takes the initiative to undertake emission reduction responsibilities, and the development of domestic carbon market will have a greater impact on China's electricity market.

\section{Data Availability}

The two time series data used to support the findings of this study have been deposited in the WIND repository and Shanghai Environment and Energy Exchange. The daily closing price of carbon price used in this study is available from Shanghai Environment and Energy Exchange (http://www.cneeex.com/); Electric Utilities Sub-Industry Index (EUI) price is selected to represent the Electricity price, which is available from Wind database.

\section{Conflicts of Interest}

The authors declare that there are no conflicts of interest.

\section{Authors' Contributions}

All authors contributed equally to this work. All authors read and approved the final manuscript.

\section{Acknowledgments}

This research was supported by the National Natural Science Foundation of China (NSFC) (Grants nos. 71273207 and 71704140) and the Soft Science Research Major Program of Shaanxi Province (no. 2011kjxx54) and The Humanities and Social Science Project of Shaanxi Bureau of Education (16JK1481).

\section{References}

[1] Anon, http://www.tanjiaoyi.com/article-23555-1.html.

[2] F. Ma, Y. Wei, and D. Huang, "Multifractal detrended crosscorrelation analysis between the Chinese stock market and 
surrounding stock markets," Physica A: Statistical Mechanics and its Applications, vol. 392, no. 7, pp. 1659-1670, 2013.

[3] M. Pal, P. Madhusudana Rao, and P. Manimaran, "Multifractal detrended cross-correlation analysis on gold, crude oil and foreign exchange rate time series," Physica A: Statistical Mechanics and its Applications, vol. 416, pp. 452-460, 2014.

[4] B. Podobnik, I. Grosse, D. Horvatić, S. Ilic, P. C. Ivanov, and H. E. Stanley, "Quantifying cross-correlations using local and global detrending approaches," The European Physical Journal B, vol. 71, no. 2, pp. 243-250, 2009.

[5] J. Theiler, S. Eubank, A. Longtin, B. Galdrikian, and J. Doyne Farmer, "Testing for nonlinearity in time series: the method of surrogate data," Physica D: Nonlinear Phenomena, vol. 58, no. 1-4, pp. 77-94, 1992.

[6] Y. Wang, Y. Wei, and C. Wu, "Cross-correlations between Chinese A-share and B-share markets," Physica A: Statistical Mechanics and its Applications, vol. 389, no. 23, pp. 5468-5478, 2010.

[7] R. Gu, H. Chen, and Y. Wang, "Multifractal analysis on international crude oil markets based on the multifractal detrended fluctuation analysis," Physica A: Statistical Mechanics and its Applications, vol. 389, no. 14, pp. 2805-2815, 2010.

[8] J. Alvarez-Ramirez, M. Cisneros, C. Ibarra-Valdez, and A. Soriano, "Multifractal Hurst analysis of crude oil prices," Physica A: Statistical Mechanics and its Applications, vol. 313, no. 3-4, pp. 651-670, 2002.

[9] J. Alvarez-Ramirez, J. Alvarez, and E. Rodriguez, "Short-term predictability of crude oil markets: A detrended fluctuation analysis approach," Energy Economics, vol. 30, no. 5, pp. 26452656, 2008.

[10] B. B. Mandelbrot, The Fractal Geometry of Nature, Freeman, San Francisco, USA, 1982.

[11] E. E. Peters, Fractal Market Analysis: Applying Chaos Theory to Investment and Economics, John Wiley \& Sons Press, New York, NY, USA, 1994.

[12] D. Kenourgios and A. Samitas, "Testing efficiency of the copper futures market: new evidence from london metal exchange," Finance, 2005.

[13] A. W. Lo, "Long-term memory in stock market prices," Econometrica, vol. 59, no. 5, pp. 1279-1313, 1991.

[14] C. Hiemstra and J. D. Jones, "Another look at long memory in common stock returns," Journal of Empirical Finance, vol. 4, no. 4, pp. 373-401, 1997.

[15] C. W. Granger, "Long memory relationships and the aggregation of dynamic models," Journal of Econometrics, vol. 14, no. 2, pp. 227-238, 1980.

[16] Y. Wang and L. Liu, "Is WTI crude oil market becoming weakly efficient over time?. New evidence from multiscale analysis based on detrended fluctuation analysis," Energy Economics, vol. 32, no. 5, pp. 987-992, 2010.

[17] W. Zhou X, "Multifractal detrended cross-correlation analysis for two nonstationary signals," Physical Review E Statistical Nonlinear \& Soft Matter Physics, vol. 77, no. 2, Article ID 066211, 2008.

[18] Y. Wang, Y. Wei, and C. Wu, "Detrended fluctuation analysis on spot and futures markets of West Texas Intermediate crude oil," Physica A: Statistical Mechanics and its Applications, vol. 390, no. 5, pp. 864-875, 2011.

[19] Y. Yuan, X.-T. Zhuang, and X. Jin, "Measuring multifractality of stock price fluctuation using multifractal detrended fluctuation analysis," Physica A: Statistical Mechanics and its Applications, vol. 388, no. 11, pp. 2189-2197, 2009.
[20] A. Serletis and I. Andreadis, "Random fractal structures in North American energy markets," Energy Economics, vol. 26, no. 3, pp. 389-399, 2004.

[21] G. J. Power and C. G. Turvey, "Long-range dependence in the volatility of commodity futures prices: Wavelet-based evidence," Physica A: Statistical Mechanics and its Applications, vol. 389, no. 1, pp. 79-90, 2010.

[22] Y. Wang, Y. Wei, and C. Wu, "Auto-correlated behavior of WTI crude oil volatilities: A multiscale perspective," Physica A: Statistical Mechanics and its Applications, vol. 389, no. 24, pp. 5759-5768, 2010.

[23] R. T. Baillie, "Long memory processes and fractional integration in econometrics," Journal of Econometrics, vol. 73, no. 1, pp. 559, 1996.

[24] P. S. Chen and Y. L. He, "Multifractal spectrum analysis of nonlinear dynamical mechanisms in China's agricultural futures markets," Physica A Statistical Mechanics \& Its Applications, vol. 389, no. 7, pp. 1434-1444, 2010.

[25] L.-Y. He and S.-P. Chen, "Nonlinear bivariate dependency of pricevolume relationships in agricultural commodity futures markets: a perspective from multifractal detrended crosscorrelation analysis," Physica A: Statistical Mechanics and its Applications, vol. 390, no. 2, pp. 297-308, 2010.

[26] B. Podobnik, D. Horvatic, A. M. Petersen, and H. E. Stanley, "Cross-correlations between volume change and price change," Proceedings of the National Acadamy of Sciences of the United States of America, vol. 106, no. 52, pp. 22079-22084, 2009.

[27] S. Shadkhoo and G. R. Jafari, "Multifractal detrended crosscorrelation analysis of temporal and spatial seismic data," The European Physical Journal B, vol. 72, no. 4, pp. 679-683, 2009.

[28] B. Podobnik, Z. Q. Jiang, W. X. Zhou, and H. E. Stanley, "Statistical tests for power-law cross-correlated processes," Physical Review E: Statistical, Nonlinear, and Soft Matter Physics, vol. 84, no. 6, Article ID 066118, 2011.

[29] B. Podobnik, Z. Jiang Q, W. Zhou X et al., "Statistical tests for power-law cross-correlated processes," Physical Review E Statistical Nonlinear \& Soft Matter Physics, vol. 84, no. 2, Article ID 066118, 2011.

[30] X. Zhuang, Y. Wei, and B. Zhang, "Multifractal detrended crosscorrelation analysis of carbon and crude oil markets," Physica A: Statistical Mechanics and its Applications, vol. 399, pp. 113-125, 2014.

[31] D. O. Cajueiro and B. M. Tabak, “The Hurst exponent over time: testing the assertion that emerging markets are becoming more efficient," Physica A Statistical Mechanics \& Its Applications, vol. 336, no. 3, pp. 521-537, 2004.

[32] G. Du and X. Ning, "Multifractal properties of Chinese stock market in Shanghai," Physica A: Statistical Mechanics and its Applications, vol. 387, no. 1, pp. 261-269, 2008. 


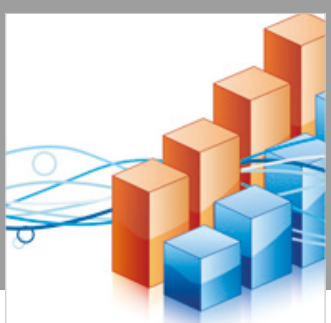

Advances in

Operations Research

\section{-n-m}
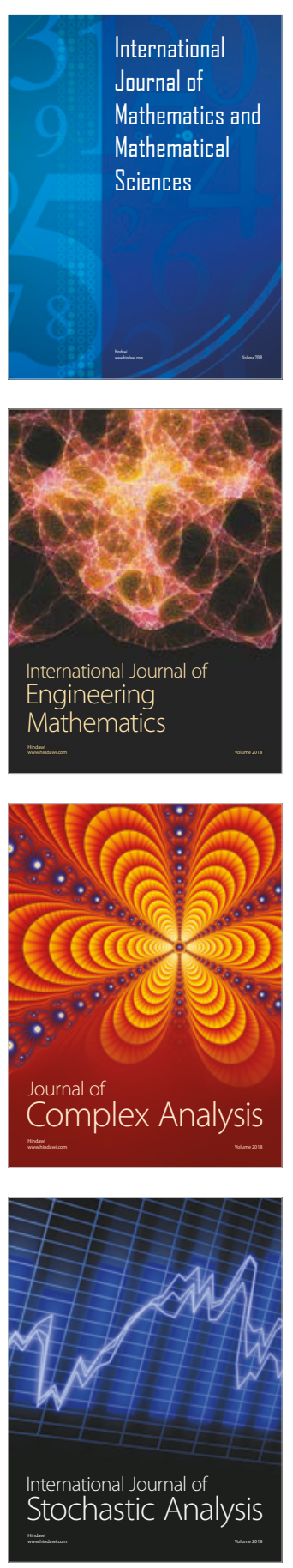
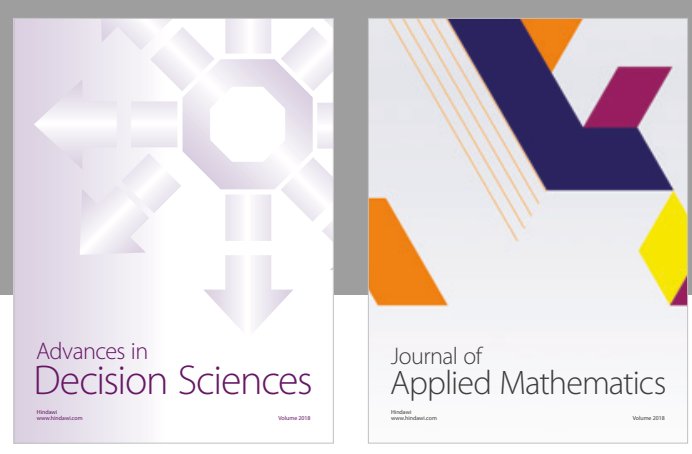

Journal of

Applied Mathematics
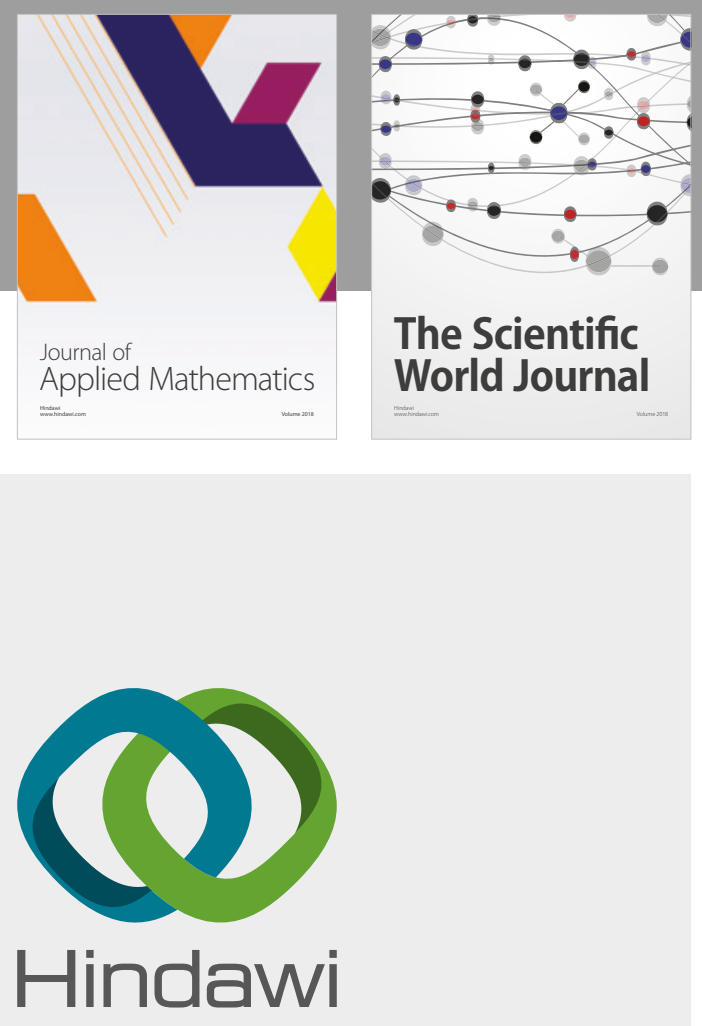

Submit your manuscripts at

www.hindawi.com

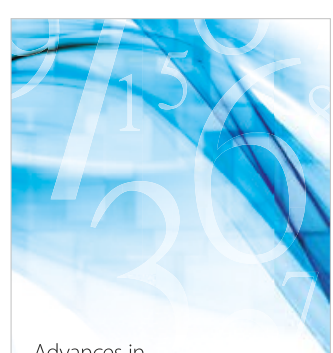

Advances in
Numerical Analysis
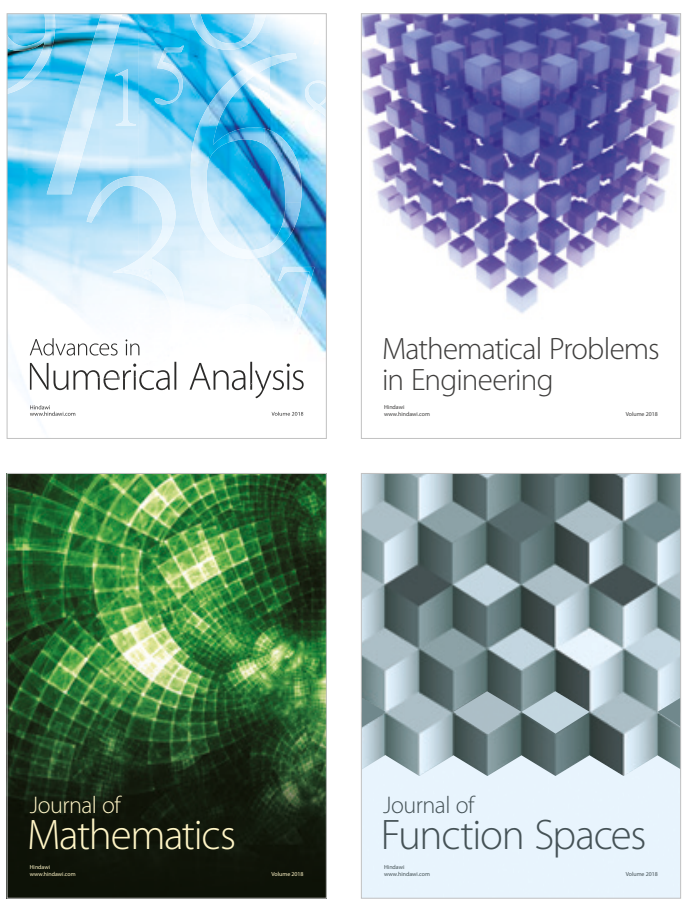

Mathematical Problems in Engineering

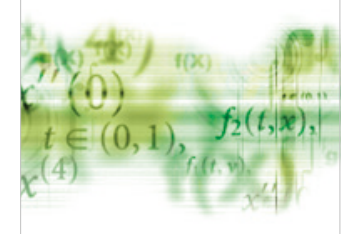

International Journal of

Differential Equations

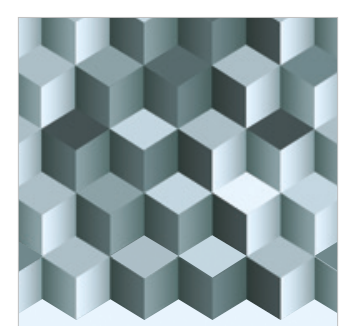

Journal of

Function Spaces

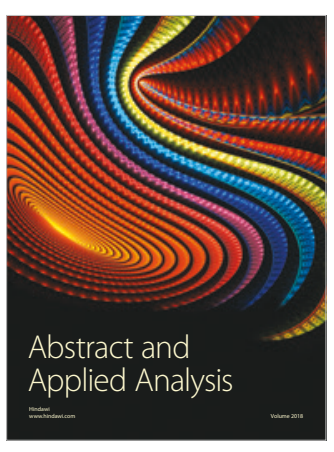

The Scientific

World Journal

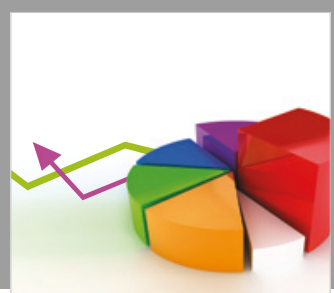

Journal of

Probability and Statistics
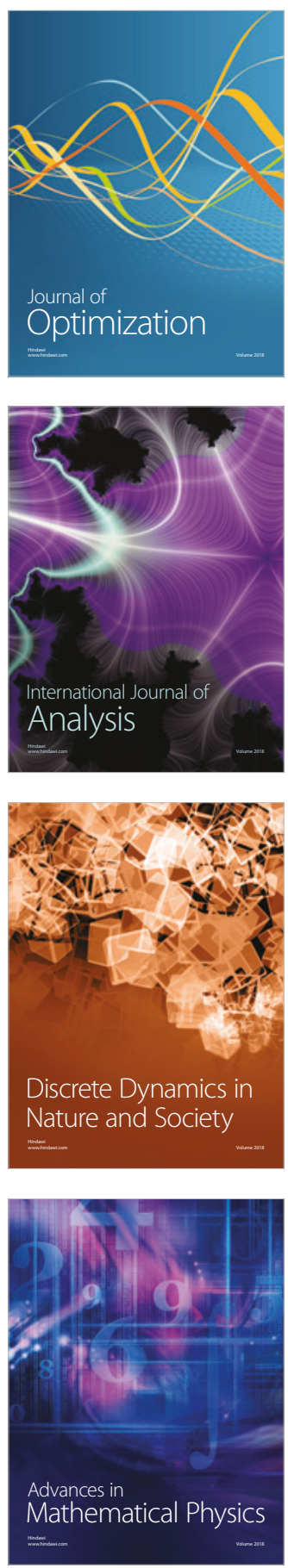